\title{
Afectación del rol maternal debido a la pandemia
} \section{Affection of the maternal role due to the pandemic}

\author{
Mirith Vásquez-Munive (iD) ${ }^{1}$, Ángela Romero -Cárdenas (iD ${ }^{2}$ \\ 1. Universidad del Magdalena. Santa Marta, Colombia. Correo: mvasquez@unimagdalena.edu.co - https://orcid.org/0000-0003-2462-4910 \\ 2. Universidad del Magdalena. Santa Marta, Colombia. Correo: aromero@unimagdalena.edu.co - https://orcid.org/0000-0003-0551-8696
}

Tipología: Artículo de investigación científica y tecnológica

Para citar este artículo: Vasquez-Munive M, Romero-Cárdenas A. Afectación del rol maternal debido a la pandemia. Duazary. 2021 septiembre; 18(3 número especial): 82-90. Doi: https://doi.org/10.21676/2389783X.4269

Recibido en abril 19 de 2021

Aceptado en julio 29 de 2021

Publicado en línea en agosto 25 de 2021

\section{Palabras clave:} maternal; teoría de enfermería pandemia; rol

\section{RESUMEN}

La capacidad de una mujer de forjar prácticas de crianza a la altura de las necesidades del hijo, se conoce como construcción del rol maternal, el cual se desarrolla dentro de un contexto que marca las necesidades de adaptación a ese rol. Actualmente la pandemia y la existencia o carencia de apoyo familiar, son las condiciones que más afectan este rol. Se presenta un estudio comparativo, utilizando muestreo tipo bola de nieve, donde se aplicó la escala adopción del rol maternal propuesta por Mercer a 23 madres con hijos menores de seis meses, 13 de ellas con apoyo familiar y 10 carentes de él. Se identificaron las características estadísticamente significativas y se llamó a las entrevistadas, para que fueran ellas las que determinaran cuales de esas características se afectaron por la pandemia. En este estudio se llegó a la conclusión que la pandemia ha afectado el rol maternal principalmente en el microsistema en ambos grupos, las madres que tienen apoyo familiar tienen mayor adopción del rol mientras las madres sin apoyo tienen menor nivel de construcción del rol y su apoyo es el uso de la tecnología. Ambos grupos sienten lejano el apoyo del sector salud.

\section{ABSTRACT}

Keywords: Pandemics; Parenting; Nursing theory.
The ability of a woman to forge parenting practices according of the needs of the child is known as the construction of the maternal role which is developed within a context that marks the adaptation needs of the role. At the present time the pandemic and the existence or lack of family support are the conditions that most affect this role. A comparative study is presented using snowball sampling, where the adoption of the maternal role scale proposed by Ramona Mercer was applied to 23 mothers with children under six months 13 of them with family support and 10 without it. When analyzing the information the characteristics that had statistically significant were taker and the interviewees were called so that they were the ones to determine which of these characteristics were affected by the pandemic. In this study it was concluded that the pandemic has affected the maternal role mainly in the microsystem in both group she mothers who have family support have a greater adoption of the role while mothers without support have a lover level of role construction and support it is the use of technology. Both groups feel distant support from the health sector. 


\section{INTRODUCCIÓN}

El rol maternal, aunque se construye a través del devenir cotidiano en el entorno familiar, se ejerce formalmente cuando la mujer tiene un hijo. Enfermería cuenta con una teoría que estudia la adopción del rol maternal formulada por Ramona Mercer, inspirada esta teoría en el modelo ecológico de Bronfrenbrenner ${ }^{1}$ y el estudio de las fases de adaptación materna en el puerperio propuestas por Rubin ${ }^{5}$ ambos autores conceden influencia decisiva al entorno para el desarrollo del rol materno debido a la relación de adaptación interactiva entra la madre y las condiciones y exigencia dinámicas del ambiente mediato e inmediato que la rodea y que condicionan la relación madre-hijo ${ }^{3,4}$.

El rol materno es un proceso complejo, donde confluyen factores cognitivos, culturales y afectivos cuya finalidad es la identificación afectiva de la diada madre-hijo, reconociendo cada uno el rol que ejerce en la vida del otro ${ }^{5}$.

Actualmente esta relación plenamente influenciada por el entorno, y el apoyo familiar se ha modificado como respuesta a la aparición en el contexto mundial de la pandemia. En este sentido, se puede afirmar que el rol parental en general y el de madre en particular ha sufrido cambios que son consecuentes con las medidas implementadas para la prevención del contagio y más allá de estas medidas, ha cambiado la forma de apoyo que pueden brindar los familiares de experiencia, y cuyo acompañamiento en este momento se realiza a través de los dispositivos tecnológicos, los mismos de que hace uso el equipo de salud para bridar asesoría y consejería para el cuidado de la madre y su neonato ${ }^{7}$.

El embarazo marca una etapa decisiva en la vida de la mujer, y por mucho que la futura madre tenga la edad y la vivencia social para tener un pensamiento abstracto y por ende anticipar situaciones problema y edificar soluciones, la maternidad de cada hijo es un hecho inédito, en muchos aspectos impredecible, cargado de emotividad, circunstancia que hace que una mujer aun con experiencia materna anterior, ante cada hijo se sienta en algunos aspectos inexperta ${ }^{8}$.

Los padres generan roles parentales dependiendo de la interacción que se establezca con el microsistema íntimo en que convive la familia y se forjan las pautas de crianza y la expresión de la afectividad, el mesosistema que es el ámbito mas cercano al círculo familiar aunque externo y el macrosistema legal y político donde se desenvuelve la dinámica familiar; de este engranaje se puede entrever que la convivencia, la cotidianidad y la construcción de roles, están plenamente influenciadas tanto por aspectos concernientes a la intimidad de los convivientes como de condiciones sociopolíticas, económicas, legales e históricas en que transcurre la maternidad ${ }^{9}$. Sin embrago es el apoyo de la familia el factor diferencial en la construcción del rol de madre.

La familia como sistema, está formada por un grupo de personas entre las cuales hay una identidad afectiva que configuran un sistema relacional y normativo determinado por sus miembros con el objeto de lograr el mayor bienestar y un nivel de protección para cada uno de ellos donde prima la protección a los miembros más pequeños.

La adopción del rol maternal es progresiva, Ramona Mercer, citada por Alvarado et $\mathrm{al}^{3}$ considera que se alcanza aproximadamente cuando la diada cumple los seis primeros meses, nunca antes y que, al cabo del año, ya la madre debe haber construido pautas de crianza y respuesta, congruentes con la personalidad de ese hijo en particular y alcanza un nivel de satisfacción y plenitud como muestra de su adopción positiva del rol. Sin embargo, llegado este momento, el rol puede estar poco construido, construido medianamente o haber una construcción positiva.

Es innegable los efectos que la pandemia tiene sobre cada uno de los aspectos de la cotidianidad individual, familiar y social lo cual indudablemente también permea la construcción de la maternidad, lo cual motivó esta investigación que tuvo por objetivo comparar cómo se ha afectado al rol 
maternal por pandemia según la disponibilidad o no de apoyo familiar.

\section{MATERIALES Y MÉTODOS}

\section{Tipo de investigación}

Estudio Descriptivo comparativo, donde se utilizó una técnica de muestreo no probabilístico tipo bola de nieve.

\section{Participantes}

A esta investigación se vincularon 23 madres, con hijos menores de seis meses, de las cuales 13 tenían apoyo familiar, constituido por el acompañamiento de la familia o la pareja y los 10 restantes fueron mujeres sin este apoyo.

\section{Técnica de recolección de la información}

A cada madre se le realizó una entrevista telefónica. Como el instrumento utilizado no está diseñado para valorar los cambios que la pandemia ha introducido a la construcción del rol matero después de aplicado, fue necesario que las madres corroboraran de las características valoradas como estadísticamente significativas, cuáles, a su criterio, habían cambiado debido a la pandemia.

\section{Instrumentos}

Se aplicó la escala adopción del rol maternal ${ }^{10}$ propuesta por Ramona Mercer, la cual está constituida por tres sistemas: macrosistema, mesosistema y microsistema, divididos a su vez en dimensiones así: el microsistema tiene seis dimensiones: Bienestar del bebé, Preocupación y protección del bebé, Estimulación del bebé, Expresiones maternales de afecto hacia el bebé, Aceptación del bebé y Contacto con el rol de madre, En total, en estas seis dimensiones que componen el microsistema, se valoran 38 características.

El mesosistema tiene las dimensiones Interacción con la pareja respecto al bebé e Interacción con la familia respecto al bebé y ellas valoran 10 características.
El macrosistema está compuesto por las dimensiones Cuidados del bebé y Conocimiento y cultura relacionados al bebé, compuestas estas dimensiones por 7 características.

La parametría del instrumento presenta los siguientes resultados ${ }^{10}$ : la validez facial para las madres fue la siguiente: alta comprensión (97\%); y para expertos: alta comprensión $(95 ; 50 \%)$, alta claridad (94;38\%) y alta precisión (93;58\%). En relevancia, el índice de validez de contenido fue de 0;97 (muy relevante) y en pertinencia, el índice de validez de contenido fue de 0;96 (muy pertinente). La validación de factores identificó diez factores que representaron el $66 \%$ de la varianza. El valor del alfa de Cronbach general fue 0;96 IC95\% (0;95-0;97). Los autores concluyen que la escala de valoración del rol maternal adaptada culturalmente para Colombia es válida y confiable para ser aplicada a madres con hijos sanos.

\section{Declaración sobre aspectos éticos}

En este estudio se respetaron las normas establecidas en la Declaración de Helsinki de 1975 y la Resolución 8430 de 1993 del Ministerio de Salud de Colombia para la investigación con seres humanos. Esto se reflejó en que las participantes consintieron expresa y voluntariamente en hacer parte del estudio y cada madre debía grabar su consentimiento antes de iniciar la aplicación del instrumento de investigación identificándose con nombre y cédula al hacerlo. Se respetó el derecho a la no participación y a dar por terminada la entrevista en el momento en que lo consideraran pertinente.

\section{RESULTADOS}

La información arrojada durante la implementación del instrumento fue analizada para identificar el sistema y las dimensiones dentro de ese sistema que arrojaran significancia estadística, para luego, introduciendo una directriz de humanización, compartir la información con las madres y aceptar que fueran ellas quienes indicaran las que en su vida tenían relevancia y que esta importancia derivara de la pandemia. 
Los resultados muestran, según se puede constatar en la tabla 1, que las madres que cuentan con el apoyo familiar, sea de la familia de ella, de la familia de él o exclusivamente de la pareja, alcanzan con mayor frecuencia niveles altos y medios de adopción del rol maternal. En ambos grupos de madres hay un número significativo que tienen nivel bajo de adopción del rol maternal, correspondiente a las madres más jóvenes y sin experiencia previa en la maternidad.

Tabla 1. Nivel de afectación del rol maternal.

\begin{tabular}{|l|l|l|l|l|l|l|}
\hline $\begin{array}{c}\text { Nivel de } \\
\text { adopción } \\
\text { el rol } \\
\text { maternal }\end{array}$ & $\begin{array}{c}\text { Con apoyo } \\
\text { del padre o } \\
\text { familia }\end{array}$ & \multicolumn{2}{|l|}{ Sin apoyo } & \multicolumn{2}{|c|}{ Total } \\
\cline { 2 - 8 } & f & $\%$ & $f$ & $\%$ & $f$ & $\%$ \\
\hline Alto & 4 & 30,76 & 1 & 10 & 5 & 21,7 \\
\hline Medio & 5 & 38.46 & 5 & 50 & 10 & 43.47 \\
\hline Bajo & 4 & 30,76 & 4 & 40 & 8 & 34,7 \\
\hline Total & 13 & 100 & 10 & 100 & 23 & 100 \\
\hline
\end{tabular}

Cuando se analiza el grupo de madres respecto a las dimensiones de adopción del rol maternal en relación con el apoyo familiar o de pareja, se encuentra significancia estadística en que el sistema más afectado es el microsistema. Como se muestra en la tabla 2, las seis dimensiones que componen el microsistema muestran afectación y entre ellas las madres indicaron que la afección se debe a la pandemia en las dimensiones preocupación y protección del bebé, aceptación del bebé estimulación del bebé, bienestar del bebé y expresiones maternales de afecto Excluyeron la dimensión contacto con el rol de madre porque consideran que ellas muestran abiertamente su condición de madre.

Las características interferidas son capacidad para salir adelante como madre, renuencia a realizar el control del niño (en la virtualidad) la lactancia es obligatoria, aunque poco segura, negación a llevarlo a la vacunación en la fecha programada.

Al analizar el mesosistema hay significancia estadística en la dimensión interacción con la pareja respecto a bebé, específicamente en las características confianza en la pareja para hacerse cargo del bebe y coordinación para satisfacer las necesidades del bebé Esta significancia estadística fue valorada por las madres como un componente de su realidad afectivamente significativo.

El macrosistema mostró significancia estadística en la dimensión cuidados del bebé, y la característica relevante fue: el personal de salud me ha enseñado los cuidados que debo tener con mi bebé. De todas las características valoradas, esta fue la más aceptada como significativa en la vida de las madres debido a que la pandemia ha impedido que haya un contacto directo con el equipo de salud lo que redunda directamente en la forma de proveer cuidado para preservar la salud de su hijo.

En la indagación sobre la afectación del rol maternal en las madres sin apoyo familiar se hallaron diferencias altamente significativas principalmente en el microsistema en las dimensiones aceptación del bebé, contacto con el rol de madre, estimulación del bebé, expresiones maternales de afecto, protección y preocupación por el bebé, resultados estos que se muestran en la tabla 3. Entre las características valoradas, no se encontró significancia estadística en la dimensión bienestar del bebé. Durante la confrontación de resultados, las madres consideraron que la estadística muestra la realidad que viven y sienten.

En el mesosistema se encontró significancia estadística en la interacción con la familia de origen respecto al bebé precisamente porque no tienen una persona significativa y cercana a quien recurrir, y este mismo hallazgo se vive en el macrosistema donde la significancia estadística esta 'en relación con el conocimiento y cultura relacionados al bebé y la vivencia muestra que la falta de apoyo directo la compensan contactando páginas y grupos en línea. 
Tabla 2 Afectación del rol maternal en mujeres con apoyo familiar según sistemas, dimensiones, características y percepción materna.

\begin{tabular}{|c|c|c|c|}
\hline Sistema & Dimensión & Característica & Significancia \\
\hline \multirow{9}{*}{ Microsistema } & \multirow{3}{*}{ Aceptación del bebé } & Me siento capaz de salir adelante con mi bebé & 0.01 \\
\hline & & $\begin{array}{l}\text { Pienso que el "control de niño sano" no es } \\
\text { necesario para mi bebé }\end{array}$ & 0.01 \\
\hline & & $\begin{array}{l}\text { Dar lactancia es más una obligación que una } \\
\text { actividad agradable }\end{array}$ & 0.02 \\
\hline & \multirow[b]{2}{*}{ Estimulación del bebé } & Mientras lo lacto me gusta acariciarlo & 0.003 \\
\hline & & $\begin{array}{l}\text { Le doy juguetes para que los toque y los } \\
\text { manipule }\end{array}$ & 0.04 \\
\hline & $\begin{array}{l}\text { Expresiones maternales de } \\
\text { afecto }\end{array}$ & Cuando le doy de lactar me gusta hablarle & 0.02 \\
\hline & Bienestar del bebé & $\begin{array}{l}\text { El momento de jugar con } \mathrm{mi} \text { bebé es } \\
\text { indispensable en i rutina }\end{array}$ & 0,024 \\
\hline & Protección y preocupación por & Hago vacunar a mi bebé en la fecha programada & 0,03 \\
\hline & el bebé & $\begin{array}{l}\text { Me preocupo porque a su alrededor no haya } \\
\text { objetos que puedan hacerle daño }\end{array}$ & 0,01 \\
\hline Mesosistema & $\begin{array}{l}\text { Interacción con la pareja } \\
\text { respecto al bebé }\end{array}$ & $\begin{array}{l}\text { Mi pareja es capaz de hacerse cargo del bebé } \\
\text { cuando yo no lo puedo hacer }\end{array}$ & 0,02 \\
\hline & & $\begin{array}{l}\text { Diariamente mi pareja busca saber cómo está el } \\
\text { bebé y coordinamos como satisfacer sus } \\
\text { necesidades }\end{array}$ & 0,026 \\
\hline Macrosistema & Cuidados del bebé & $\begin{array}{l}\text { El personal de salud me ha enseñado los } \\
\text { cuidados que debo tener con el bebé }\end{array}$ & 0,005 \\
\hline
\end{tabular}

Tabla 3. Afectación del rol maternal en mujeres sin apoyo familiar según sistemas, dimensiones y características y percepción materna.

\begin{tabular}{|c|c|c|c|}
\hline Sistema & Dimensión & Característica & Significancia \\
\hline \multirow{10}{*}{ Microsistema } & \multirow{3}{*}{ Aceptación del bebé } & Me siento capaz de salir adelante con mi bebé & 0,03 \\
\hline & & $\begin{array}{l}\text { El control de niño sano no es importante para mi } \\
\text { bebé }\end{array}$ & 0,01 \\
\hline & & $\begin{array}{l}\text { Cuando cosquilleo a mi bebé observo una } \\
\text { repuesta de alegría en él }\end{array}$ & 0,02 \\
\hline & \multirow[t]{2}{*}{$\begin{array}{l}\text { Contacto con el rol de } \\
\text { madre }\end{array}$} & $\begin{array}{l}\text { Cuando mi bebé responde a mis llamados o } \\
\text { caricias yo lo abrazo o lo felicito }\end{array}$ & 0,001 \\
\hline & & Me agrada masajear la espalda de mi bebé & 0,2 \\
\hline & \multirow[t]{2}{*}{ Estimulación del bebé } & $\begin{array}{l}\text { Le doy objetos para que los toque y aprenda a } \\
\text { manipularlos }\end{array}$ & 0,3 \\
\hline & & Trato que mi bebé acaricie mi rostro & 0,01 \\
\hline & $\begin{array}{l}\text { Expresiones maternales de } \\
\text { afecto }\end{array}$ & Cuando doy de lactar a mi bebé me gusta hablarle & 0,026 \\
\hline & \multirow[t]{2}{*}{$\begin{array}{l}\text { Protección y preocupación } \\
\text { por el bebé }\end{array}$} & Hago vacunar a mi bebé en la fecha programada & 0,03 \\
\hline & & $\begin{array}{l}\text { Cada vez que mi bebé llora me preocupo por } \\
\text { saber que necesita }\end{array}$ & 0,1 \\
\hline \multirow[t]{2}{*}{ Mesosistema } & \multirow[t]{2}{*}{$\begin{array}{l}\text { Interacción con la familia de } \\
\text { origen respecto al bebé }\end{array}$} & $\begin{array}{l}\text { La forma como crío a mi bebé es parecida a como } \\
\text { me criaron a mi }\end{array}$ & 0,02 \\
\hline & & $\begin{array}{l}\text { Las cosas que aprendí sobre el cuidado de mi } \\
\text { bebé me las enseñaron las personas que me } \\
\text { criaron }\end{array}$ & 0,028 \\
\hline Macrosistema & $\begin{array}{l}\text { Conocimiento y cultura } \\
\text { relacionados al bebé }\end{array}$ & $\begin{array}{l}\text { Constantemente me informo por programas en } \\
\text { línea de cómo cuidar a mi bebé }\end{array}$ & 0,05 \\
\hline
\end{tabular}


En ambos grupos hay afectación de los tres sistemas coincidiendo en las características de las dimensiones del microsistema estimulación y expresiones de afecto.

En la aceptación del bebé las madres sin apoyo familiar no hicieron referencia a la lactancia, mientras que, si referenciaron las manifestaciones de alegría cuando interactúan los dos, también aceptan más la cercanía con su hijo porque al estar más alejados de otras personas tienden a acercarse y no ven esta cercanía como una amenaza a la salud del bebé.

Al valorar la protección del bebé las madres con apoyo familiar se preocupan por los objetos que hay en el entorno para que no resulten dañinos para el niño, mientras que las madres que carecen de este apoyo centran su preocupación en cómo interpretar el llanto del hijo en términos de entender que les pasa o que necesitan. En este caso está afectada la misma dimensión con características diferentes. En el mesosistema las madres con apoyo buscan que el padre se involucre en el cuidado, mientras las mujeres sin apoyo acuden al referente de cómo fueron criadas y pautas de comportamiento que han visto a su alrededor, de igual forma que buscan consejo y apoyo haciendo uso de la tecnología.

\section{DISCUSIÓN}

Esta investigación fue motivada por las constantes quejas de las madres con respecto a las dificultades durante toda la gestación y en su ejercicio de la maternidad inducidos por la pandemia. Considerando que las madres con apoyo familiar tienden a construir con mayor facilidad y rapidez el rol materno ${ }^{11}$, el objetivo fue comparar esta adopción del rol en madres con y sin apoyo familiar, dejando que las mismas madres, indicaran cuales, de las características y dimensiones estadísticamente significativas, eran también percibidas por ellas como significantes en su proceso de crianza y por ende en el desarrollo y adquisición del rol materno.

Los resultados muestran sincronía entre la medida estadística y el sentir de las madres en su cotidianidad. Sin embargo, es innegable que la pandemia ha alejado al binomio madre hijo de las instituciones de salud y ha acercado el cuidado a la tecnología. La atención en salud no presencial es un cambio valorado como ineficaz, pero necesario en las actuales condiciones. Esta medida emergente constituye un recurso del macrosistema como parte de la adecuación de los cuidados en tiempos pandémicos realidad que no es ajena a ningún grupo con necesidades especiales de cuidado como bien afirma la Organización Mundial de la Salud ${ }^{12}$.

Los hallazgos reportan que las madres que cuentan con apoyo, construyen el rol en uh nivel más alto, aunque se evidencia que aun con apoyo permanente y positivo, hay casos de madres que muestran bajo nivel de construcción del rol, resultado que sería lo esperado entre madres que no cuentan con apoyo. Estos resultados son congruentes con lo encontrado por Garrido y Marchan ${ }^{13}$ en su estudio cuando concluye que el apoyo puede provenir de la pareja, de la familia de cualquiera de los dos, o de cualquier persona afectivamente significativa para la mujer, pero siempre va a constituir un factor favorecedor para la maternidad, aunque no excluye que aun con apoyo familiar el rol puede no ser muy alto, debido a otros factores propios del microsistema como la propia relación con el hijo y el manejo del estrés.

El modelo de adopción del rol maternal establece que la madre, de acuerdo al contexto es sensible a las señales del hijo, desarrollando la capacidad de introducir cambios y modulando su conducta hasta lograr la adaptación a la situación propuesta y en este caso es la situación pandémica que ha introducido formas distintas de vivir la maternidad y el cuidado del hijo, como lo referencian López et al ${ }^{14}$ en su estudio, donde evidencian que uno de los riesgos que ha inducido la pandemia es el estrés y temor excesivos de la madre lo que la lleva a reaccionar de manera desproporcionada ante las señales del hijo como el llanto, la madre lo vive con desesperación y no ajusta una conducta positiva sino que transita por la duda y el error.

Las capacidades de la madre van desarrollándose en la medida en que vaya viviendo experiencias con su 
hijo, va desarrollando confianza, autonomía y adquiriendo el rol de madre. Esta conducta se ve favorecida por la interacción con personas significativas en la vida, es decir por el acompañamiento de la pareja y la familia Las condiciones de aislamiento social imponen alejamiento familiar y por lo tanto desfavorecen la adquisición de rol materno. Este aspecto es notorio en la presente investigación cuando para las entrevistadas es significativo tener la confianza en que llegarán a ser unas buenas madres. Al respecto el fondo de población de las Naciones Unidas UNFPA $^{15}$, reconoce que uno de los riesgos de a pandemia es la inestabilidad y la desconfianza que en las mujeres genera y que se refleja en la poca confianza para vivir plenamente sus derechos sexuales y reproductivos y desempeñarse como madre.

En ambos grupos de madres, la lactancia ha pasado a ser una obligación más que una actividad agradable por el miedo a contagiar al bebé y aunque la lactancia fisiológicamente se constituye en un momento de alta empatía física y mental con el hijo, donde las hormonas confieren gran sensibilidad a la glándula mamaria, la madre, actualmente, vive este momento con desasosiego y ambigüedad, por eso no lo acarician, no le hablan y hacen de momento de la lactancia una instrumentación netamente nutritiva. Estos hallazgos son coincidentes con los referenciados por Montero y $\operatorname{Caparros}^{16}$, que en su revisión sobre el tema de lactancia y covid, demostraron que las madres no se sienten seguras de la inocuidad de la lactancia debido a la cercanía con su hijo y que, en aras de privilegiar la salud del recién nacido, se debe alentar a la madre a que tome una decisión fundamentada en la evidencia, pero con respeto de su sentir.

Al hablar del mesosistema, autores como Marín et $\mathrm{al}^{17}$, hacen referencia a la importancia de las relaciones familiares en la formación del hijo y la adquisición del rol, en términos de apoyo a la crianza, aseveración que se acerca a lo encontrado entre las madres entrevistadas. Estas conclusiones son congruentes con lo que perciben las mujeres en esta investigación ambos grupos de madres señalaron el gran valor que constituye el contar con el apoyo de la pareja y/o la familia para proveer cuidado efectivo, mientras que las madres que no cuentan con este apoyo refieren que ellas, a falta de una figura familiar y cercana que les indique a partir de la experiencia cual es la mejor manera de afrontar cada situación, se refugian en replicar las formas de crianza con las cuales convivieron en su familia de origen.

El macrosistema, para las madres con apoyo familiar está representado por el personal de salud, al cual en muy pocas oportunidades pueden recurrir, pero cuyo apoyo añoran. Al respecto Becerra-Bula et $a^{18}$, manifiestan que la familia es un factor facilitador asociado a la adquisición de pautas de crianza positivas.

Para las madres sin apoyo, la información necesaria para forjar unas buenas pautas de crianza y cuidado proviene de la tecnología. Para ambos grupos, el apoyo social y familiar es percibido como fundamental, y la falta de él significa que no se promueven conductas necesarias $y$ no se construyen lazos que promuevan bienestar psicológico y confianza en el desempeño del rol. Estas consideraciones también fueron obtenidas por Ortiz et $a l^{19}$ y Espinal et $a l^{20}$ en sus estudios, donde referencian el valor del apoyo familiar y social para todo el proceso de crianza, el acompañamiento que requiere la familia.

\section{CONCLUSIÓN}

-La pandemia ha afectado la construcción del rol maternal, impactando en los tres sistemas, siendo más influyente en el microsistema.

-Las madres que conviven con la pareja o la familia o con ambos, tienen garantías para construir lazos de apoyo emocional, instrumental e informativo para sobrellevar la crianza con mayor facilidad, aunque la información que reciben en cuanto al devenir de la pandemia las sumerja en un ambiente de incertidumbre que tiene como consecuencia directa las variaciones en las conductas de cercanía con el hijo como medio de prevención hacia el contagio.

- Las madres sin apoyo familiar intentan subsanar la carencia recurriendo al personal de salud que en este momento puede brindar apoyo emocional instrumental e informativo con las limitaciones que 
impone el distanciamiento social y el uso de la tecnología, percibida esta conducta como insuficiente y lejana.

\section{AGRADECIMIENTOS}

Agradecemos a las madres que muy amablemente dispusieron de su tiempo para atender nuestras llamadas, a la universidad del Magdalena por su apoyo y al comité científico del congreso internacional de salud que hicieron posible la publicación de esta investigación.

\section{DECLARACION SOBRE CONFLICTOS DE INTERESES}

Las autoras declaran que no existió conflicto de intereses en esta investigación

\section{CONTRIBUCIÓN DE LOS AUTORES}

Primer autor: diseño metodológico, trabajo de campo, análisis y redacción;

Segundo autor: diseño metodológico, trabajo de campo, análisis y redacción

\section{REFERENCIAS BIBLIOGRÁFICAS}

1.Torrico E. et al. El modelo ecológico de Bonfrenbrenner como marco teórico de la psicooncología. Anales de psicología [revista en la Internet]. 2002 [citado 2021 Jun 18]; 18(1); 45-59. Disponible en: https://www.um.es/analesps/v18/v18_1/0318_1.pdf"

2. Moreno C, Peralta A, Velásquez N. Convertirse en madre durante la adolescencia: transiciones del rol materno. Inv. en enf. Imag. y Dllo. [revista en la Internet]. 2020 [citado $2021 \mathrm{Jul}$ 21]; 22: 129-142. DOI: https://doi.org/10.11144/Javeriana.ie22.cmda

3. Alvarado L. et al., Adopción del Rol Maternal de la teorista Ramona Mercer al cuidado de enfermería binomio madre-hijo: Reporte de caso en la unidad Materno Infantil. Cuidarte. [revista en la Internet]. 2011 [citado 2021 Mayo 5]; 3(4):195 -203. Disponible http://www.scielo.org.co/pdf/cuid/v2n1/v2n1a15. pdf

4. Chjnchilla E. Trabajo con una familia, un aporte desde la orientación familiar. Actualidades investigativas en ed. [revista en la Internet]. 2015 [citado 2021 Abril 5]; 15(1):1 -27. Disponible en: https://www.redalyc.org/pdf/447/44733027039.pd $f$

5. Rubin, R. Maternal identity and the maternal experience. New York: Springer. 1984. Disponible en https://journals.Iww.com/ajnonline/Citation/1984/ 12000/MATERNAL_IDENTITY_AND_THE_MATERNA L_EXPERIENCE.30.aspx

6. Organización Mundial de la Salud. Pautas para el cuidado de la salud perinatal y de la niñez. New York, E.U. Disponible en: http://www.codajic.org/sites/www.codajic.org/file s/Pautas\%20para\%20el\%20cuidado\%20de\%20la\%2 Osalud\%20perinatal\%20y\%20de\%20la\%20ni\%C3\%B 1ez.pdf

7. Lagos R, Suárez MC, Torres A, Sánchez M, Murat I. Proceso de una maternidad deseada en pandemia: cartografía de emociones desencadenadas. Rev psicol. UNC. [revista en la Internet]. 2020 [citado 2021 Mayo 6]; 5(2):71 -88. Disponible en: https://revistas.unc.edu.ar/index.php/aifp/article/v iew/31072/31715

8. Baena-Antequera $F$, et al. Atención del embarazo durante la pandemia del covid-19, ¿un impulso para el cambio? Rev Esp. Sal. Publica. [revista en la Internet]. 2020 [citado 2021 Abril 5]; 94(6): 18-26. Disponible en: https://www.mscbs.gob.es/biblioPublic/publicacio nes/recursos_propios/resp/revista_cdrom/VOL94/ C_ESPECIALES/RS94C_202011157.pdf

9. Sánchez-Rivera M. Construcción social de la maternidad, el papel de las mujeres en la sociedad. [revista en la Internet]. 2016 [citado 2021 Abril 8]; 32 (13): 921-953. Disponible en: https://www.redalyc.org/pdf/310/31048483044.pd $f$ 
10. Vargas-Porras C, et al. Adaptación cultural, validez y confiabilidad de la escala de adopción del rol materno en Colombia. Rev Cien e Innovación en salud. [revista en la Internet]. 2020; [citado 2021 Julio 10]; e70:1-19. Disponible en: DOI:10.17081/innosa.70

11. Molina de Juan, M. Cuidado de los hijos en tiempo de pandemia, apuntes para repensar la experiencia argentina. Rev. Act. Jur. Iberoam. [revista en la Internet]. 2020 [citado 2021 Julio 10]; 12(2), 190-201. Disponible en: http://www.revistaaji.com/wp-

content/uploads/2020/05/19._Mariel_Molina_pp._ 190-201.pdf

12. Romero G. El CGE y las asociaciones de matronas resuelven las dudas de las embarazadas durante la pandemia. Diario Enfermero. España. 2020 [citado 2021 Abril 30]; Disponible en: https://diarioenfermero.es/el-cge-y-lasasociaciones-dematronas-resuelven-las-dudas-delas-embarazadas-durante-la-pandemia/

13. Garrido M. Marchan M. Adopción del rol materno en madres adolescentes primerizas en relación con el grupo de convivencia. Rev Psic. Troj. [revista en la Internet]. 2011 [citado 2021 Junio 6]; 13(1):11 -28. Disponible en: http://revistas.ucv.edu.pe/index.php/R_PSI/article/ view/306

14. López P, Pantoja L, Melia M, Utreras M, Vrgara C. Revisión de los riesgos maternos y perinatales en tiempos de covid. Un desafío para la matronería. Rev. Chil. Obs Ginec. 2020; 85 (1): 131-158. Disponible en: https://www.scielo.cl/scielo.php?script=sci_arttext \&pid=S0717-75262020000700018

15. Fondo de población de las naciones unidas. Los riegos de la pandemia para el desarrollo de los derechos sexuales y reproductivos de las mujeres. Disponible en https://oig.cepal.org/sites/default/files/folleto_ssy r_esp_0.pdf

16. Montero E, Caparros RA. Covid 19 en embarazo, lactancia materna y transmisión vertical. Anales del sistema sanitario de Navarra. [revista en la Internet].
2020 [citado 2021 Julio 10]; 43 (2): 27-39. Disponible en:

https://recyt.fecyt.es/index.php/ASSN/article/view $/ 81528$

17. Marín-Iral M. del P, Quintero-Córdoba P, RiveraGómez S. Influencia de las relaciones familiares en la primera infancia. Poiésis, [revista en la Internet]. 2019 [citado 2021 Mayo 10]; (36), 164-183. Disponible en DOI: https://doi.org/10.21501/16920945.319

18. Becerra-Bula F, Rocha-Calderón L., Fonseca-Silva D., Bermúdez-Gordillo L. El entorno familiar y social de la madre como factor que promueve o dificulta la lactancia materna. Rev. Fac med. [revista en la Internet]. 2017 [citado 2021 Mayo 8]; 63(2), 217$227 . \quad$ Disponible en: https://www.redalyc.org/articulo.oa?id=57636352 4006

19. Ortiz-Félix R., Cárdenas-Villarreal V, Flores-Peña R. Modelo del rol materno en la alimentación del lactante: una teoría de rango medio. Index de enf. [revista en la Internet]. 2017 [citado 2021 Junio 8]; 25(3), 193-206. Disponible en https://scielo.isciii.es/scielo.php?script=sci_arttext \&pid $=\mathrm{S} 1132-$

12962016000200009\#: :text=El\%20modelo\%20de \%20rol\%20materno,el\%20estado\%20nutricional\%2 Odel\%20lactante.

20. Espinal I, Gimeno A, González F. El enfoque sistémico en los estudios sobre la familia. Rev UASD. [revista en la Internet]. 2016; [citado 2021 Julio 10]; 18 (5): 48-61. Disponible en: https://www.uv.es/jugar2/Enfoque\%20Sistemico.p df 